and apoptosis analysis was performed by flow cytometry. Gene expression in mock and shHOXD9 SKG-I cells was analyzed by microarray.

Results: HOXD9 inhibition suppressed P105 promoter activity and E6/E7 gene expression in HPV18 positive cervical cancer cell line. Cell cycle analysis indicated that HOXD9 blocking increased the ratio of G1 phase cells, decreased cell proliferation and enhanced apoptosis. HOXD9 suppression did not change the P53 gene level, but restored the P53 protein expression. Furthermore, the expression of MCM2 and PCNA, the target genes of transcription factor E2F, was decreased in HOXD9 inhibited cells. Finally, promoter assay and chromatin immunoprecipitation assay showed that HOXD9 directly bound to the P105 promoter and activated its function.

Conclusion: HOXD9 played an important role in cell proliferation and immortalization by regulating the P105 promoter in HPV18 positive cervical cancer. These findings indicated that HOXD9 might be a promising therapeutic target in cervical cancer.

Poster (C11)

Preinvasive Disease of Cervix, Vulva, and Vagina

https://doi.org/10.3802/jgo.2021.32.S1.C11

\section{The overtreatment rate of "see and treat" approach in high-grade squamous cervical cytology at Ramathibodi hospital}

\footnotetext{
Watanya Rongthongaram, Chuenkamon Charakorn," Sikarn Satitniramai, Arb-Aroon Lertkhachonsuk

Department of Obstetrics and Gynecology, Faculty of Medicine, Ramathibodi Hospital, Mahidol University, Bangkok, Thailand (cheunkamonc@hotmail.com)
}

Objective: To evaluate an overtreatment rate of "see and treat" (S\&T) approach in patients with high-grade squamous intraepithelial lesion (HSIL) cervical cytology and identify the clinical factors associated with the overtreatment.

Methods: Patients with HSIL cytology undergoing colposcopy and loop electrosurgical excision procedure (LEEP) in a single visit or S\&T approach between January 2005 to December 2019 at colposcopic clinic, Ramathibodi hospital were retrospectively reviewed. Overtreatment was defined as the pathological examination of LEEP specimen reported cervical intraepithelial neoplasia 1 or normal results. The overtreatment rate and complication event from LEEP was explored. Clinical factors affecting the overtreatment were analyzed with a logistic regression model.

Results: There were 220 cases identified. The overtreatment rate was $11.4 \%$. The surgical complications composed of 3 cases with hemorrhage (1.36\%) and 9 cases with infection (4.09\%). In univariable analysis, factors associated with the overtreatment were present cytology and colposcopic diagnosis with p-value of 0.006 and 0.001 , respectively. In multivariable analysis, present cytologic results of non-HSIL compared to HSIL and colposcopic diagnosis (low-grade lesion or normal finding compared to high grade lesion or cancer) were presented adjusted odds ratio of 13.81 and 3.58 (95\% confidence interval $[\mathrm{CI}]=1.23-155.20 ; \mathrm{p}=0.033$ and 95\% CI=1.32-9.74; $\mathrm{p}=0.013$, respectively).

Conclusion: The overtreatment rate in S\&T approach was $11.4 \%$. The independent factors associated with overtreatment rate were the present cytology and colposcopic diagnosis.

Poster (C12)

Cervical Cancer

https://doi.org/10.3802/jgo.2021.32.S1.C12

\section{To compare the pre-operative and post operative staging in early stages of cervical cancer on the basis of changes made in FIGO 2018 staging}

Richi Khandelwal, "Bhagyalakshmi Nyak, Jita Parija, Manoranjan Mahapatra, Ashok Padhy, Janmejeya Mohapatra, Sushil Giri

Acharya Harihar Post Graduate Institute of Cancer, Cuttack, India (richi_kh@yahoo.com)

Objective: To compare the pre-operative and post operative staging in early stages of cervical cancer on the basis of changes made in International Federation of Gynecology and Obstetrics (FIGO) 2018 staging.

Methods: An observational hospital-based study was conducted in which 57 cases were included who were surgically staged. Cases were re-staged on the basis of the 2018 FIGO staging and clinicoradiological and surgico-pathological factors were considered. Data was collected to identify patients who were upstaged or downstage, need of adjuvant treatment were analysed.

Results: The $86.2 \%$ patients had undergone preoperative imaging. Ultrasound was the most commonly done follow by magnetic resonance imaging and computed tomography scan. The 20 patients were upstaged and 5 patients were downstage on the basis of final histopathological report. The $27 \%$ patients were given adjuvant treatment. The most common factor was the change in tumor size followed by parametrical invasion and the least common was lymphovascular space invasion.

Conclusion: The new FIGO 2018 staging system led to an upstage in $35.08 \%$ patients and helps in better delineation of treatment both primary and adjuvant, if needed. 